\title{
ALGEBRAIC PROPERTY OF ROUGH IMPLICATION BASED ON INTERVAL STRUCTURE
}

\author{
Xue Zhan-ao, He Hua-can and Ma Ying-cang \\ School of Computer Science, Northwestern Polytechnical University, Xi'an 7l0072, China
}

\begin{abstract}
Due to the shortage of rough implication in [4] [6], rough set and rough implication operators are redefined by using interval structure in [7], the shortages have been e improved. We have investigated the characteristics of the rough implication, and also point out that the good logic property of the rough implication in [7]. In this paper, we will study the algebraic properties of the rough implication in depth.
\end{abstract}

Key words: Rough Logic, Algebraic Property, Rough Implication, Approximation Spaces

\section{INTRODUCTION}

Rough set theory, introduced by Zdzislaw Pawlak in the early $1980 \mathrm{~s}^{[1-3]}$, is a new mathematical tool to deal with many problems such as vagueness, uncertainty, incomplete data and reasoning. Now there are lots of papers about rough logic idea and its abroad application ${ }^{[1-9]}$, but some rough implication operators exist defects, for instance, $B^{c} \rightarrow A^{c}=A \rightarrow B$ doesn't hold in [4], $A \rightarrow A$ is not Theorem in [5,6], etc.. In order to eliminate those defects we redefine rough set system, and new rough operators such as intersection, union, complement and implication are expressed by using interval structure in [7]. The characteristics of this implication were investigated, and logic properties of rough implication were pointed out in [7]. Further, we will study the algebraic properties of the rough implication in this paper. 


\section{ROUGH SET THEORY}

Definition2.1 Let $U$ be the universe set and $R$ be an equivalent relation on $U$. A pair

$(U, R)$ is called an approximate space. If $X \subseteq U$ is an arbitrary set, then two approximations are formally defined as follows:

$$
\underline{X}=\left\{x \mid x \in U,[x]_{R} \subseteq X\right\}, \quad \bar{X}=\left\{x \mid x \in U,[x]_{R} \cap X \neq \phi\right\} .
$$

Where $[x]_{R}$ is an equivalent class containing $x . \underline{X}$ is called lower approximation of $X, \bar{X}$ is called upper approximation of $\bar{X}$. The approximate set $X$ lies between its lower and upper approximations: $\quad X \subseteq X \subseteq \bar{X}$.

We get $-\bar{X} \subseteq-X \subseteq-\underline{X}$, where, $Z \subseteq U$ and $-Z$ is the complement of $Z$ in $U$.

For each $X \subseteq U$, a rough set is a pair $\langle\underline{X}, \bar{X}\rangle$. We denote the empty set $\phi$ by $\langle\phi, \bar{\phi}\rangle=\langle\phi, \phi\rangle$, the universe set $U$ by $\langle\underline{U}, \bar{U}\rangle=\langle U, U\rangle$ and the power set of $U$ by $\Re(U)$.

Definition 2.2 Let $A, B \in \mathfrak{R}(U)$, the inclusion relation of two rough sets is defined by $A \widetilde{\subset} B$ if and only if $\bar{A} \subseteq \bar{B}$ and $\underline{A} \subseteq \underline{B}$;

The equivalent relation of two rough sets is defined by

$A=B$ if and only if $\bar{A}=\bar{B}$ and $\underline{A}=\underline{B}$.

Definition 2.3 The intersection of two rough sets $A$ and $B$ is a rough set in approximate space, and is defined by $A \cap B=\langle\underline{A} \cap \underline{B}, \bar{A} \cap \bar{B}\rangle$,

The union of two rough sets is a rough set in approximate space, and is defined by $A \cup B=\langle\underline{A} \cup \underline{B}, \bar{A} \cup \bar{B}\rangle$,

The complement of $A$ is a rough set in approximate space, and is defined by $A^{c}=\langle-\bar{A},-\underline{A}\rangle$,

The pseudo complement of $A$ is a rough set in approximate space, and is defined by $A^{*}=\langle-\underline{A},-\underline{A}\rangle$,

Where $X \subseteq U,-X$ is the complement of $X$ in $\mathrm{U}$.

Theorem 2.4 Suppose $A, B \in \mathfrak{R}(U)$, then

$\underline{A \cap B}=\underline{A} \cap \underline{B}, \overline{A \cap B} \subseteq \bar{A} \cap \bar{B}$

$\overline{A \cup B} \supseteq \underline{A} \cup \underline{B}, \overline{A \cup B}=\bar{A} \cup \bar{B}$.

Proof. Theorem 2.4 follows from [1] [3] and [8] [9].

Theorem 2.5 If $A^{c}$ is the complement of $A$ in $\mathrm{U}, A^{*}$ is the pseudo complement of $A$ in $\mathrm{U}$, then
(1) $A^{c} \subseteq A^{*}$;
(2) $A^{* *} \subseteq A^{c *}$
(3) $A^{c} \cup A^{*}=A^{*}, A^{c} \cap A^{*}=A^{c}$;
(4) $A^{c * c}=A^{c * *}=\langle-\bar{A},-\bar{A}\rangle$;
(5) $A^{c c *}=A^{* c *}=A^{* * *}=A^{* * c}=A^{* c c}=A^{*}$;
(6) $A^{c c c}=A^{c}$;
(7) $A^{c * c^{* *}}=A^{c *}$.

Proof. Theorem 2.5 can be proved easily from Definition 2.3.

Theorem 2.6 Let $A, B \in \mathfrak{R}(U)$, then,
$(A \cap B)^{c}=A^{c} \cup B^{c}$;
$(A \cup B)^{c}=A^{c} \cap B^{c}$;
$(A \cap B)^{*}=A^{*} \cup B^{*}$;
$(A \cup B)^{*}=A^{*} \cap B^{*}$.

Proof. Theorem 2.6 is easy to be proved by Definition 2.3. 


\section{ALGEBRAIC PROPERTIES OF ROUGH IMPLICATION}

We redefine the implication operator in [7], which to improve the shortage of

rough implication in [4] [6]. In this section, we will directly cite the definition implication operator $\rightarrow$, and will investigate its algebraic properties.

Definition 3.1 Let $m n g(\varphi)=\langle\underline{A}, \bar{A}\rangle, m n g(\psi)=\langle\underline{B}, \bar{B}\rangle, m n g(\beta)=\langle\underline{C}, \bar{C}\rangle$, and $m n g$ is a bijection, for any $\varphi, \psi, \beta, 0,1 \in P$, we have

$$
\begin{aligned}
& m n g(\varphi \wedge \psi)=\langle\underline{A} \cap \underline{B}, \bar{A} \cap \bar{B}\rangle ; \quad m n g(\varphi \vee \psi)=\langle\underline{A} \cup \underline{B}, \bar{A} \cup \bar{B}\rangle ; \\
& m n g\left(\varphi^{c}\right)=\langle-\bar{A},-\underline{A}\rangle ; \quad m n g\left(\varphi^{*}\right)=\langle-\underline{A},-\underline{A}\rangle ; \quad m n g(0)=\langle\phi, \phi\rangle ; \\
& m n g\left(\varphi^{c *}\right)=\langle\bar{A}, \bar{A}\rangle ; \quad m n g\left(\varphi^{c * c}\right)=\langle-\bar{A},-\bar{A}\rangle ; \quad m n g(1)=\langle U, U\rangle . \\
& (\varphi \vee \psi)^{c}=\varphi^{c} \wedge \psi^{c} ; \quad(\varphi \wedge \psi)^{c}=\varphi^{c} \vee \psi^{c} ; \quad(\varphi \vee \psi)^{*}=\varphi^{*} \wedge \psi^{*} ; \\
& (\varphi \wedge \psi)^{*}=\varphi^{*} \vee \psi^{*} ; \quad \varphi^{c * c}=\varphi^{c * *} ; \quad \varphi^{c c c}=\varphi^{c} ; \\
& \varphi^{c * c^{*}}=\varphi^{c *} ; \quad \varphi^{c c^{*}}=\varphi^{* c^{*}}=\varphi^{* * *}=\underline{\varphi}^{* * c}=\varphi^{* c c}=\varphi^{*} \text {. } \\
& \text { (I) } \\
& m n g(\varphi \rightarrow \psi)=m n g\left(\varphi^{c} \vee \psi \vee\left(\varphi^{*} \wedge \psi^{c *}\right)\right)=\langle-\bar{A} \cup \underline{B} \cup(\bar{B} \cap-\underline{A}),-\underline{A} \cup \bar{B}\rangle
\end{aligned}
$$

Theorem $3.2\left(P, \vee, \wedge,{ }^{c}, 0,1\right)$ is a boundary lattice.

Proof. Theorem 3.2 is easy to prove from definition 3.1 and [7].

Theorem 3.3 Let $A, B \in \mathfrak{R}(U)$, the following are equivalent:

(1) $A \rightarrow B=\langle-\bar{A} \cup \underline{B} \cup(\bar{B} \cap-\underline{A}),-\underline{A} \cup \bar{B}\rangle$;

(2) $A \rightarrow B=\langle(-\underline{A} \cup \underline{B}) \cap(-\bar{A} \cup \bar{B}),-\underline{A} \cup \bar{B}\rangle$.

Proof. Theorem 3.3 is easy to be proved from (I).

Proposition 3.4 Suppose $\left(P, \vee, \wedge,{ }^{c}, 0,1\right)$ is called a boundary lattice which is inverse ordered involution, and $\rightarrow$ is rough implication operator, the following are satisfied:

$$
\begin{array}{lr}
\text { (IA.1) } \varphi \rightarrow(\psi \rightarrow \beta)=\psi \rightarrow(\varphi \rightarrow \beta) & \text { (IA.2) } \varphi \rightarrow \varphi=1 \\
\text { (IA.3) } \varphi \rightarrow \psi=\psi^{c} \rightarrow \varphi^{c} & \text { (IA.4) if } \varphi \rightarrow \psi=\psi \rightarrow \varphi=1, \\
\varphi=\psi & \\
\text { (IA.5) } & \\
\varphi \vee \psi \rightarrow \beta=(\varphi \rightarrow \beta) \wedge(\psi \rightarrow \beta) & \text { (IA.6) } \varphi \wedge \psi \rightarrow \beta=(\varphi \rightarrow \beta) \vee(\psi \rightarrow \beta)
\end{array}
$$

Proof. The formulas can be proved by Theorem 3.3 and (I).

Proof of (IA.1)

$$
\begin{aligned}
& \varphi \rightarrow(\psi \rightarrow \beta)=\varphi^{c} \vee(\psi \rightarrow \beta) \vee\left(\varphi^{*} \wedge(\psi \rightarrow \beta)^{c^{*}}\right) \\
& =\varphi^{c} \vee\left(\psi^{c} \vee \beta \vee\left(\psi^{*} \wedge \beta^{c^{*}}\right)\right) \vee\left(\varphi^{*} \wedge\left(\psi^{c} \vee \beta \vee\left(\psi^{*} \wedge \beta^{c^{*}}\right)\right)^{c *}\right) \\
& =\varphi^{c} \vee \psi^{c} \vee \beta \vee\left(\psi^{*} \wedge \beta^{c *}\right) \vee\left(\varphi^{*} \wedge\left(\psi^{*} \vee \beta^{c *} \vee\left(\psi^{*} \wedge \beta^{c *}\right)\right)\right) \\
& =\varphi^{c} \vee \psi^{c} \vee \beta \vee\left(\psi^{*} \wedge \beta^{c *}\right) \vee\left(\varphi^{*} \wedge \psi^{*}\right) \vee\left(\varphi^{*} \wedge \beta^{c *}\right) \vee\left(\varphi^{*} \wedge \psi^{*} \wedge \beta^{c *}\right) \\
& \psi \rightarrow(\varphi \rightarrow \beta)=\psi^{c} \vee(\varphi \rightarrow \beta) \vee\left(\psi^{*} \wedge(\varphi \rightarrow \beta)^{* *}\right) \\
& =\psi^{c} \vee\left(\varphi^{c} \vee \beta \vee\left(\varphi^{*} \wedge \beta^{c *}\right)\right) \vee\left(\psi^{*} \wedge\left(\varphi^{c} \vee \beta \vee\left(\varphi^{*} \wedge \beta^{c *}\right)\right)^{c *}\right) \\
& =\psi^{c} \vee \varphi^{c} \vee \beta \vee\left(\varphi^{*} \wedge \beta^{c^{*}}\right) \vee\left(\psi^{*} \wedge\left(\varphi^{*} \vee \beta^{c^{*}} \vee\left(\varphi^{*} \wedge \beta^{c *}\right)\right)\right)
\end{aligned}
$$




$$
\begin{aligned}
& =\psi^{c} \vee \varphi^{c} \vee \beta \vee\left(\varphi^{*} \wedge \beta^{c *}\right) \vee\left(\psi^{*} \wedge \varphi^{*}\right) \vee\left(\psi^{*} \wedge \beta^{c *}\right) \vee\left(\psi^{*} \wedge \varphi^{*} \wedge \beta^{c *}\right) \\
& =\varphi^{c} \vee \psi^{c} \vee \beta \vee\left(\psi^{*} \wedge \beta^{c *}\right) \vee\left(\varphi^{*} \wedge \psi^{*}\right) \vee\left(\varphi^{*} \wedge \beta^{c *}\right) \vee\left(\varphi^{*} \wedge \psi^{*} \wedge \beta^{c *}\right) \\
& \text { Hence, } \varphi \rightarrow(\psi \rightarrow \beta)=\psi \rightarrow(\varphi \rightarrow \beta)
\end{aligned}
$$

Proof of (IA.2) Obviously, $\varphi \rightarrow \varphi=1$

Proof of (IA.3)

$$
\begin{aligned}
& \varphi^{c} \rightarrow \psi^{c}=\varphi^{c c} \vee \psi^{c} \vee\left(\varphi^{c *} \wedge \psi^{c c^{*}}\right)=\psi^{c} \vee \varphi \vee\left(\psi^{*} \wedge \varphi^{c *}\right) \\
& \psi \rightarrow \varphi=\psi^{c} \vee \varphi \vee\left(\psi^{*} \wedge \varphi^{c *}\right)
\end{aligned}
$$$$
\text { Hence, } \varphi \rightarrow \psi=\psi^{c} \rightarrow \varphi^{c} \text {. }
$$

Proof of (IA.4)

Because of $A \rightarrow B=\langle(-\underline{A} \cup \underline{B}) \cap(-\bar{A} \cup \bar{B}),-\underline{A} \cup \bar{B}\rangle \quad A \rightarrow B=U \quad$ iff $\mathrm{A} \rightarrow \mathrm{B}=\langle(-\underline{\mathrm{A}} \cup \underline{\mathrm{B}}) \cap(-\overline{\mathrm{A}} \cup \overline{\mathrm{B}}),-\underline{\mathrm{A}} \cup \overline{\mathrm{B}}\rangle=\langle\underline{\mathrm{U}}, \mathrm{U}\rangle$ iff $-\underline{\mathrm{A}} \cup \underline{\mathrm{B}}=\overline{\mathrm{U}},-\overline{\mathrm{A}} \cup \overline{\mathrm{B}}=\mathrm{U},-\underline{\mathrm{A}} \cup \overline{\mathrm{B}}=\mathrm{U}$

iff $A \subseteq B$ and $\bar{A} \subseteq \bar{B}$ iff $A \widetilde{\subset B}$.

Analogously we have $B \rightarrow A=U$ iff $B \widetilde{\subset} A$.

Hence, (IA.4) is proved.

Proof of (IA.5)

$(\varphi \rightarrow \beta) \wedge(\psi \rightarrow \beta)=\left(\varphi^{c} \vee \beta \vee\left(\varphi^{*} \wedge \beta^{c *}\right)\right) \wedge\left(\psi^{c} \vee \beta \vee\left(\psi^{*} \wedge \beta^{c *}\right)\right)$ $=\left(\varphi^{c} \wedge \psi^{c}\right) \vee\left(\varphi^{c} \wedge \beta\right) \vee\left(\varphi^{c} \wedge \psi^{*} \wedge \beta^{c *}\right) \vee\left(\beta \wedge \psi^{c}\right) \vee \beta \vee\left(\beta \wedge \psi^{*} \wedge \beta^{c *}\right)$

$\vee\left(\varphi^{*} \wedge \psi^{c} \wedge \beta^{c *}\right) \vee\left(\beta \wedge \varphi^{*} \wedge \beta^{c *}\right) \vee\left(\varphi^{*} \wedge \psi^{*} \wedge \beta^{c^{*}}\right)$

$=\left(\varphi^{c} \wedge \psi^{c}\right) \vee \beta \vee\left(\varphi^{*} \wedge \psi^{*} \wedge \beta^{c^{*}}\right)$

$(\varphi \vee \psi) \rightarrow \beta=(\varphi \vee \psi)^{\mathrm{c}} \vee \beta \vee\left((\varphi \vee \psi)^{*} \wedge \beta^{\mathrm{c} *}\right)=\left(\varphi^{\mathrm{c}} \wedge \psi^{\mathrm{c}}\right) \vee \beta \vee\left(\varphi^{*} \wedge \psi^{*} \wedge \beta^{\mathrm{c*}}\right)$

Hence, $\varphi \vee \psi \rightarrow \beta=(\varphi \rightarrow \beta) \wedge(\psi \rightarrow \beta)$.

Proof of (IA.6)

$(\varphi \wedge \psi) \rightarrow \beta=(\varphi \wedge \psi)^{c} \vee \beta \vee\left((\varphi \wedge \psi)^{*} \wedge \beta^{c *}\right)=\left(\varphi^{c} \vee \psi^{c}\right) \vee \beta \vee\left(\left(\varphi^{*} \vee \psi^{*}\right) \wedge \beta^{c *}\right)$

$=\left(\varphi^{c} \vee \psi^{c}\right) \vee \beta \vee\left(\varphi^{*} \wedge \beta^{c *}\right) \vee\left(\psi^{*} \wedge \beta^{c *}\right)$

$(\varphi \rightarrow \beta) \vee(\psi \rightarrow \beta)=\left(\varphi^{c} \vee \beta \vee\left(\varphi^{*} \wedge \beta^{c *}\right)\right) \vee\left(\psi^{c} \vee \beta \vee\left(\psi^{*} \wedge \beta^{c *}\right)\right)$

$=\left(\varphi^{c} \vee \psi^{c}\right) \vee \beta \vee\left(\varphi^{*} \wedge \beta^{c *}\right) \vee\left(\psi^{*} \wedge \beta^{c *}\right)$

Hence, $\varphi \wedge \psi \rightarrow \beta=(\varphi \rightarrow \beta) \vee(\psi \rightarrow \beta)$.

The poor is complete.

Proposition 3.5 Suppose $\left(P, \vee, \wedge,{ }^{c}, 0,1\right)$ is called a boundary lattice which is inverse ordered involution, and $\rightarrow$ is rough implication operator which is expressed by interval structure, then $\quad(\varphi \rightarrow \psi) \rightarrow \psi \neq(\psi \rightarrow \varphi) \rightarrow \varphi$

Proof.

$(\varphi \rightarrow \psi) \rightarrow \psi=\left(\varphi^{c} \vee \psi \vee\left(\varphi^{*} \wedge \psi^{c *}\right)\right)^{c} \vee \psi \vee\left(\left(\varphi^{c} \vee \psi \vee\left(\varphi^{*} \wedge \psi^{c *}\right)\right)^{*} \wedge \psi^{c^{*}}\right)$

$=\left(\varphi \wedge \psi^{c} \wedge\left(\varphi^{* c} \vee \psi^{c * c}\right)\right) \vee \psi \vee\left(\left(\varphi^{c *} \wedge \psi^{*} \wedge\left(\varphi^{* *} \vee \psi^{c * *}\right)\right) \wedge \psi^{c *}\right)$

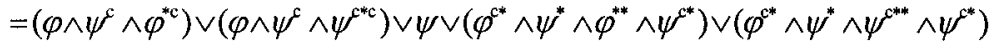
$=\left(\psi^{c} \wedge \varphi^{* c}\right) \vee\left(\varphi \wedge \psi^{c * c}\right) \vee \psi \vee\left(\psi^{*} \wedge \varphi^{* *} \wedge \psi^{c *}\right)$

Analogously, $(\psi \rightarrow \varphi) \rightarrow \varphi=\left(\varphi^{c} \wedge \psi^{* c}\right) \vee\left(\psi \wedge \varphi^{c * c}\right) \vee \varphi \vee\left(\varphi^{*} \wedge \psi^{* *} \wedge \varphi^{c *}\right)$

Hence, $(\varphi \rightarrow \psi) \rightarrow \psi \neq(\psi \rightarrow \varphi) \rightarrow \varphi$ (except for $\varphi=\psi$ ).

Remark If $\left(P, \vee, \wedge,{ }^{c}, 0,1\right)$ is lattice implicative algebra ${ }^{[11-13]}$, then it is satisfied Proposition 3.4 (IA.1) (IA.6) and $(x \rightarrow y) \rightarrow y=(y \rightarrow x) \rightarrow x$. Since the equivalence of lattice implicative algebra is normal $F I$-algebra ${ }^{[11}$, 
14], the operator $\rightarrow$ is not satisfied $(x \rightarrow y) \rightarrow y=(y \rightarrow x) \rightarrow x$. Hence, $\left(P, \vee, \wedge,{ }^{c}, 0,1\right)$ is not lattice implicative algebra, but it is $F I$-algebra ${ }^{[11,14]}$.

\section{CONCLUSION}

The study of rough implication operators is the emphasis and difficulty in the field of rough logic. Due to definition the shortages of the rough implication operator in [4] [6], we can not imply $B^{c} \rightarrow A^{c}=A \rightarrow B$ in [4], i.e. the inversely negative proposition and original proposition are not equivalent, and $\mathrm{A} \rightarrow \mathrm{A}$ isn't Theorem in $[5,6]$, etc... We redefine the rough intersection, rough union, rough complement and rough implication operator from the view of interval structure, which their relations and properties have been investigated in [7]. In this paper, we_study the algebraic properties of the rough implication in a deep way, and also point out that $\left(P, \vee, \wedge,{ }^{c}, 0,1\right)$ is not lattice implicative algebra, but it is $F I$-algebra, because the formula $(x \rightarrow y) \rightarrow y=(y \rightarrow x) \rightarrow x$ doesn't hold.

\section{ACKNOWLEDGEMENTS}

This paper is supported by the National Natural Science Foundation (No.60273087) and Beijing Nature Science Foundation of China (No.4032009).

\section{REFERENCES}

1. Pawlak Z. Rough Sets. International Journal of Computer and Information Sciences, 1982, 11: 341 356.

2. Pawlak Z. Rough Sets-Theoretical Aspects of Reasoning about Data. Kluwer Academic Publishers. Dordrecht, 1991.

3. Pawlak Z. Vagueness and Uncertainty: A Rough Set Perspective, Computational Intelligence. 1995, 11:227 232.

4. Duntsch I. Logic for Rough Sets. Theoretical Computer Sciences, 1997, 179:427 436.

5. E.Orlowska Reasoning about Vague Concepts. Bull. Pol. Ac: Mathematics, 1987, 35:643 652.

6. Ceng H L. Rough set theory and Application. Chongqing University Press, Chongqing, 1998(in Chinese).

7. Xue $\mathrm{Z}$ A. and He H C. Rough Implication. Journal of Computer and Sciences (in Chinese). 2003, 30(11):18 20.

8. Zhang Wenxiu etc... Rough set theory \& method. Science Press, Beijing, 2001.7 (in Chinese).

9. Zhu F. He H C. The Axiomatization of the Rough Set. Journal of Computer (in Chinese). 2000, 23(3):330 333. 
10. Wong S.K.M. Wang L.S. Yao Y.Y. On Modeling Uncertainty with Interval Structures. International Journal of Computational Intelligence. 1995, 11 (2):406 426.

11. Wang G J. MV-Algebras, BL-Algebras, $\mathrm{R}_{0^{-}}$-Algebras, and Multiple-Valued Logic. Fuzzy Systems and Mathematics (in Chinese).2002, 16(2):1 15.

12. Chang C C. Algebraic analysis of many-valued [J]. Trans.Amer.Math.Soc.1958, 88:467 490.

13. Xu Y. Lattice implicative algebra. Journal of Southwest Jiao tong University, 1993, 28(1): 20 26.

14. Jakubi'k J. Direct product decomposition of MV-algebras. Czechoslovak Mathematical Joural, 1994, 44:725 793. 